\title{
Fas stimulation of T lymphocytes promotes rapid intercel- lular exchange of death signals via membrane nanotubes
}

\author{
Peter D Arkwright ${ }^{1,2}$, Francesca Luchetti ${ }^{2,3}$, Julien Tour ${ }^{2}$, Charlotte Roberts ${ }^{2}$, Rahna Ayub ${ }^{2}$, Ana P Morales ${ }^{2}$, \\ José J Rodríguez ${ }^{2}$, Andrew Gilmore ${ }^{2}$, Barbara Canonico ${ }^{3}$, Stefano Papa ${ }^{3}$, Mauro Degli Esposti ${ }^{2}$ \\ ${ }^{I}$ University of Manchester, Royal Manchester Children's Hospital, Manchester; ${ }^{2}$ Faculty of Life Sciences, University of Manches- \\ ter, Oxford Road, Manchester, M13 9PT, United Kingdom; ${ }^{3}$ Department of Human, Environmental and Nature Science, University \\ of Urbino, Italy
}

The Fas/CD95 surface receptor mediates rapid death of various cell types, including autoreactive $T$ cells with the potential for triggering autoimmunity. Here, we present novel aspects of Fas signalling that define a 'social' dimension to receptor-induced apoptosis. Fas stimulation rapidly induces extensive membrane nanotube formation between neighbouring $T$ cells. This is critically dependent on Rho GTPases but not on caspase activation. Bidirectional transfer of membrane and cytosolic elements including active caspases can be observed to occur via these nanotubes. Nanotube formation and intercellular exchanges of death signals are defective in $T$ lymphocytes from patients with autoimmune lymphoproliferative syndrome harbouring mutations in the Fas receptor. We conclude that nanotubemediated exchanges constitute a novel form of intercellular communication that augments the propagation of death signalling between neighbouring $\mathrm{T}$ cells.

Keywords: Fas, intercellular communication, membrane nanotubes, $\mathrm{T}$ cells

Cell Research (2010) 20:72-88. doi: 10.1038/cr.2009.112; published online 22 September 2009

\section{Introduction}

Apoptosis can be defined as a physiological mechanism by which redundant or diseased cells are removed without inducing an inflammatory response. Aside from inducing activation of caspases critical for cell execution [1], apoptosis stimulates major alterations in the cell membranes [2]. In the immune system, death receptors mediate physiological cell death via the 'extrinsic' pathway, named so because it follows receptor ligation with cell-surface proteins such as FasL (CD95L/CD178).

Correspondence: Mauro Degli Esposti

Tel: +44-1612755447; Fax: +44-1612755762

E-mail: mauro.esposti@manchester.ac.uk

Abbreviations: ALPS (autoimmune lymphoproliferative syndrome); CdTXB (toxin B of Clostridium difficile); CFP (cyan fluorescent protein); CFSE (carboxyfluorescein succinimidyl ester); CtxB (Cholera toxin subunit B); HPA (Helix pomatia agglutinin); RB (modified Ringer buffer); Rho-IETD-bis (rhodamine110-carbonyl-Ile-Glu-Thr-Asp-bisamide); WGA (wheat germ agglutinin); YFP (yellow fluorescent protein); z-VAD (benzyloxycarbonyl-Val-Ala-Asp-fluoromethylketone)

Received 16 April 2009; revised 9 June 2009; accepted 25 June 2009; published online 22 September 2009
Transmission of signals inside the cell is accomplished by trimerization of these receptors, which then drives the activation of apical caspase- 8 and other caspases leading to cellular autodigestion [3]. Fas/CD95, the prototypic death receptor, also undergoes rapid internalization after triggering $[2,4]$.

Fas-mediated apoptosis is important for peripheral deletion of activated T lymphocytes [1]. Autoimmune lymphoproliferative syndrome (ALPS) is a rare genetic disorder of humans resulting from defects in the apoptotic pathway of T lymphocytes [5], most commonly caused by mutations in the cytoplasmic domain of the Fas receptor (type Ia ALPS $[6,7]$ ). The condition is associated not only with benign and less commonly malignant T-cell lymphoproliferation, but also with various autoimmune phenomena, particularly autoimmune cytopenias. Other aspects of T-cell function are usually intact. ALPS provides a very useful model for studying the importance of Fas in triggering the biochemical as well as membrane changes induced by the apoptotic cascade [2]

Membrane nanotubes are dynamic plasma membrane extensions forming channels of communication between neighbouring cells, especially of the immune system [8- 
10]; they have not previously been implicated in apoptosis. Here, we report that Fas signalling increases intercellular exchanges along membrane nanotubes connecting different cells. Our results suggest that these membrane nanotubes allow bi-lateral transfer of membrane and cytosolic components promoting the propagation of apoptosis within a cell population, in a way that is defective in $\mathrm{T}$ cells from ALPS patients.

\section{Results}

Fas signalling increases cell conjugation leading to differential death rates

We found that Fas signalling rapidly promoted intercellular contacts, increasing conjugation of Jurkat $\mathrm{T}$ cells (Figure 1A). The orientation of conjugation was twofold higher than the expected random distribution in relation to the position of the Golgi region labelled with fluorescent derivatives of Cholera toxin B (CtxB, Figure 1A cf. $[11,12])$. In particular, the Golgi region was usually orientated at the opposite side of cell conjugation (Supplementary information, Figure S1A). We then addressed the question as to whether increased cell conjugation affected Fas-induced cell death and its propagation to bystander T cells.

We undertook detailed analysis of $T$ cells attached to cover slips and two early indicators of cell death: caspase activation and membrane blebbing, which correlate well with DNA fragmentation [13], the terminal signature of apoptosis (Figure 1B). Conjugated cells often exhibited asymmetric rates of apoptosis, with one cell of the conjugate displaying indicators of apoptosis, while the other showed none (Figure 1B and 1C). We then distinguished conjugated from isolated non-conjugated cells in timelapse images, noting the asymmetrical distribution of incipient Fas-induced apoptosis. The time-course of caspase-8 activation, monitored with a fluorogenic substrate for this apical caspase [13], showed that Fas stimulation with its cognate ligand induced more rapid apoptosis in conjugated than isolated cells (Figure 1C and 1D). The same difference between conjugated and non-conjugated cells was observed by following the time-course of cell blebbing (Figure 1E). Similar results were obtained by automated scoring of the staining for active caspase- 3 (Supplementary information, Figure S1B).

\section{Fas signalling enhances the formation of interconnecting nanotubes}

Our results indicated that Fas stimulation led to an intriguing (and previously unsuspected) asymmetry of death propagation depending upon cell conjugation. What type of cell contact could be responsible for this asymmetry? Extending our analysis to high-resolution images obtained with fluorescence deconvolution, we noted that Jurkat and primary $\mathrm{T}$ cells were often connected to each other via filamentous protrusions of different length and size (Figure 2). These protrusions, contrary to cell conjugates, increased in number by increasing cell density (Supplementary information, Figure S1C) and generally displayed features characteristic of membrane (or tunneling) nanotubes, a recently described form of intercellular communication $[8,9,14,15]$. In particular, membrane nanotubes sprouted at different levels along the vertical plane of Fas-stimulated T cells and, contrary to filopodia, hovered away from the adhesion surface (Figure 2B and Supplementary information, Figure S2).

Membranes nanotubes usually carried volleys of fluorescent lectins such as Helix pomatia agglutinin (HPA) or wheat germ agglutinin (WGA), mixed with membranes containing either transmembrane proteins like CD81 (Figure 2A) or glycosyl phosphatidylinositol-anchored proteins like CD59 (Supplementary information, Figure S2A). However, some nanotubular connections were poorly stained with lectins but enriched in membrane lipids or CD59 (Figure 2C and Supplementary information, Figure S2). Of note, mitochondrial organelles were not detected in membrane nanotubes either before or after Fas stimulation (Figure 2C and data not shown). In contrast, connecting nanotubes invariably contained actin filaments, which formed structural elements resembling 'buttress' scaffoldings, increasing the spatial complexity of nanotubular connections (Figure 2D).

Fas activation with FasL or the agonist antibody $\mathrm{CH}-$ 11 equally augmented the development of connecting nanotubes, which peaked at 20 to $30 \mathrm{~min}$ (Figure 3A and 3B). Similar time-courses were obtained by following live cell imaging (Figure 3A, Supplementary information, Figures $\mathrm{S} 2 \mathrm{~F}$ and $\mathrm{S} 3 \mathrm{~A}$ ) or by analysing fixed cells (Figure 3B), even though more nanotubes were seen in live cells owing to their dynamic and transient nature (cf. [8, 14]). Fas-induced nanotube formation occurred before overt activation of caspases, since it was comparable in cells with no caspase- 8 activity (Supplementary information, Figure S2B) and after inhibition with the general caspase inhibitor benzyloxycarbonyl-Val-AlaAsp-fluoromethylketone (z-VAD) (Figure 3). A similar induction of nanotubes was produced by acute treatment with TRAIL, another ligand of ubiquitous death receptors (Supplementary information, Figures S2E and S3B).

Agents that disrupted actin polymerization and assembly blocked the formation of membrane nanotubes, consistent with the fundamental role of actin in sustaining these structures $[8,14]$. The general inhibitor of actinregulating Rho GTPases, toxin B of Clostridium difficile 
A

Cell conjugation

$\square$ Unteated

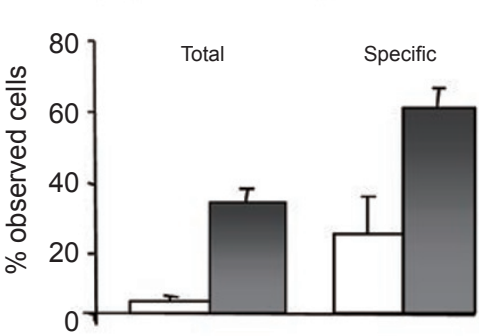

C

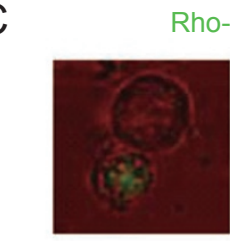

32
Rho-IETD-bis

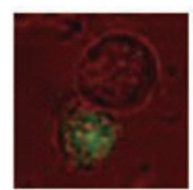

34
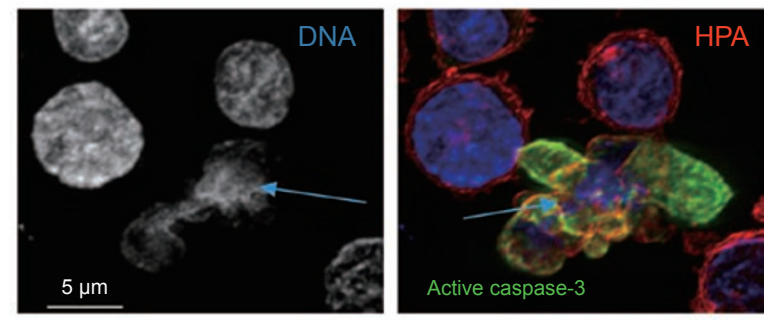

Brightfield

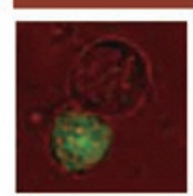

38

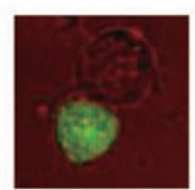

42

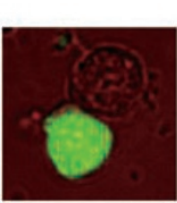

46

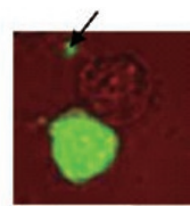

50

Time (min) after FasL

D

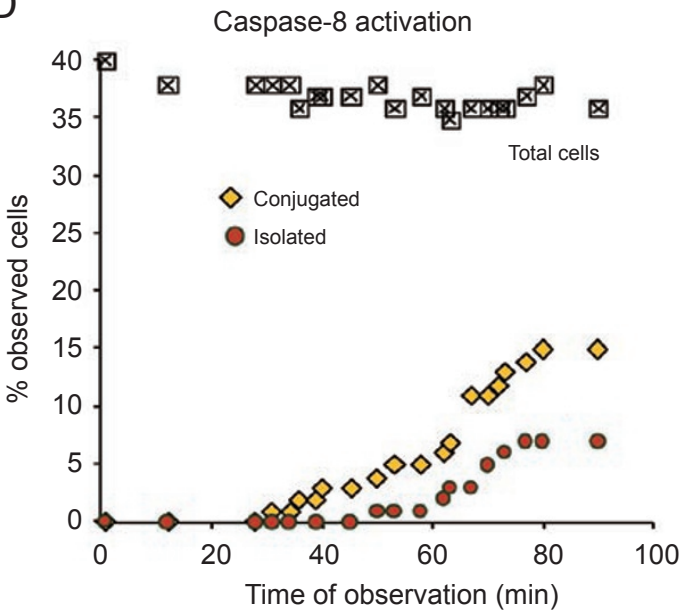

E

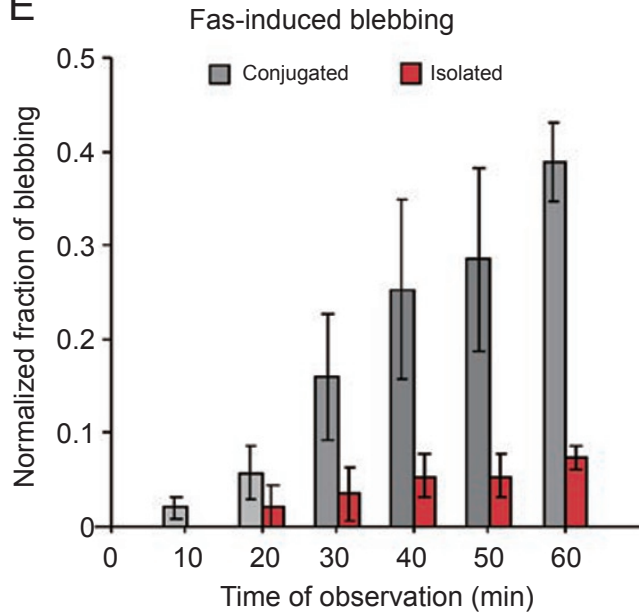

Figure 1 Fas stimulation increases cell conjugation leading to asymmetric death. (A) Fas stimulation increases cell conjugation. The histograms on the left represent the average percentage of couplings in $>2000$ Jurkat T cells from $n=5$ separate experiments with cell density varying from 2 to $5 \times 10^{6}$ per $\mathrm{ml}$. The histograms on the right represent the average frequency of 'specific polarization', which was evaluated by four independent scorers in images of cells labelled with markers of the Golgi region like CtxB $[11,12]$ (Supplementary information, Figure S1A). Blind scoring counted conjugates in which one cell had its Golgi region clearly at the opposite side of the junction with the other cell, a configuration that increased after FasL treatment from the expected frequency of around $30 \%$ in untreated cells. Statistical differences between FasL-treated and untreated cells were highly significant $(P<0.02$, Mann-Whitney). (B) Representative image combining the three markers for apoptosis used here to determine the level of cell death: nuclear fragmentation with the blue Hoechst dye, activation of caspase-3 with a specific antibody (with a green secondary) and membrane blebbing with red HPA. The blebbing cell showing nuclear fragmentation and diffuse caspase activation is conjugated with a cell showing no evident sign of incipient apoptosis. (C) The sequence of frames shows a cell conjugate displaying asymmetric fluorescence of Rhodamine110-IETD-bisamide (10 $\mu \mathrm{M}$, a substrate for caspase-8 $[13,37])$ in live cell images of Jurkat cells treated with FasL. Bright field images are coloured red for contrast. The arrow indicates a surface membrane cluster (microparticle?) apparently lying at the extreme periphery of the top cell that showed delayed caspase activation. (D) The time-course of caspase activation was evaluated by time-lapse images like those in $\mathbf{C}$ using one $\times 100$ field, representative of three other. Cell conjugates showing asymmetric levels of green fluorescence as in $\mathbf{C}$ (diamonds) were distinguished from isolated green cells (circles). (E) Time-course of cell blebbing was evaluated from bright field images scoring isolated and conjugated cells as in D. Results from $n=4$ separate experiments showed statistically significant differences between isolated and conjugated blebbing cells after 30 min of Fas stimulation ( $P$ $=0.03$, Mann-Whitney). 

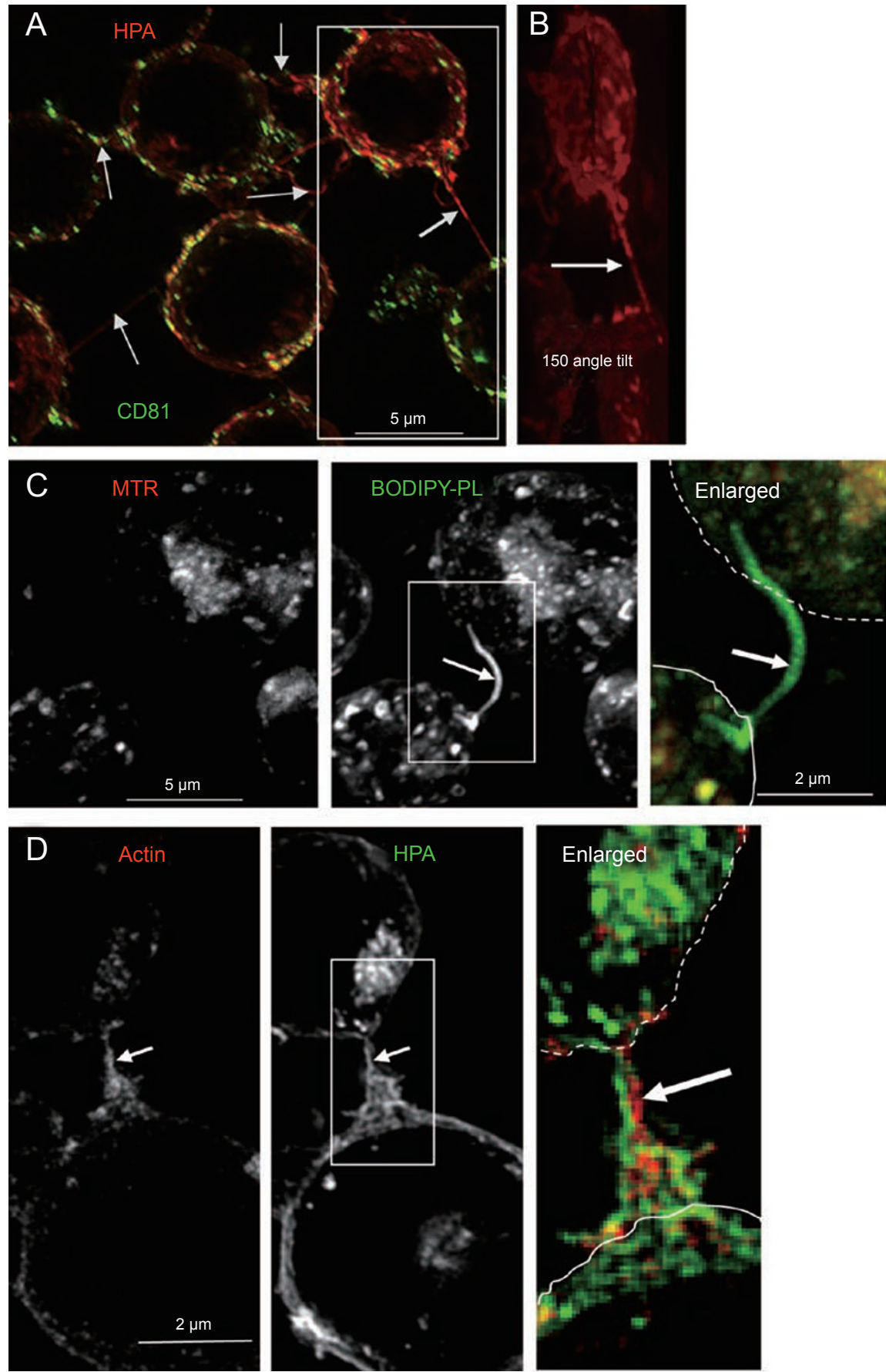

Figure 2 Fas signalling increases the formation of connecting membrane nanotubes. (A) The image shows the cumulative z-projection of 29 sections of cells, which were surface stained with red HPA after internalization of FITC-conjugated antiCD81 as described earlier [13]. Arrows point to the various connecting nanotubes that emerge at different equatorial levels along the $z$ axis. Deconvolved images were taken with $a \times 100$ objective. (B) The image is slightly enlarged from the box area in $\mathbf{A}$ and tilted by an angle of 150 degrees to better show the long connecting nanotube sprouting from the central part of the top cell. Only the surface red staining is shown. (C) Cells were incubated with 50 nM Mitotracker ${ }^{\circledR}$ red (MTR) and then with liposomes containing fluorescent BODIPY-phosphatidylcholine (Molecular Probes) plus BODIPY-monolysocardiolipin (Degli Esposti, unpublished) mixed with non-fluorescent membrane phospholipids. After equilibration and washing, fluorescent lipids were predominantly endocytosed and accumulated in nanotubular protrusions (arrow), similar to carbocyanine lipophilic dyes (cf. [21]). (D) After 30 min of treatment with the Fas-activating antibody $\mathrm{CH}-11$, Jurkat cells were fixed, permeabilized with $0.2 \%$ saponin and then incubated with $10 \mu \mathrm{M}$ Texas red-conjugated phalloidin (red, to stain F-actin) and then stained with $2 \mu \mathrm{g} / \mathrm{ml}$ of AlexaFluor488-conjugated HPA (green). Arrow indicates the large connecting nanotube enriched in actin (enlarged). 
A Live cell images

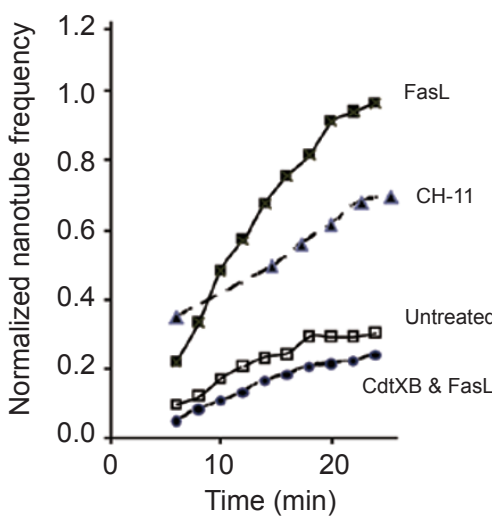

B $\quad$ Fixed cell images

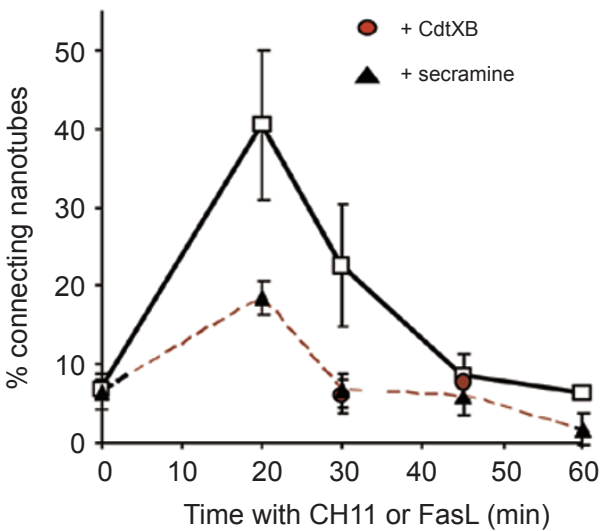

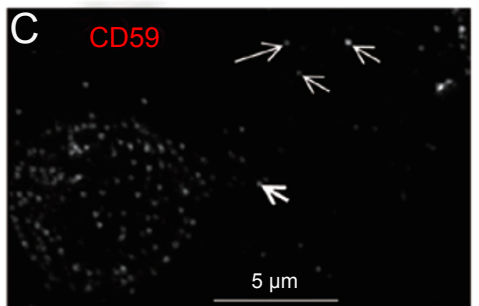
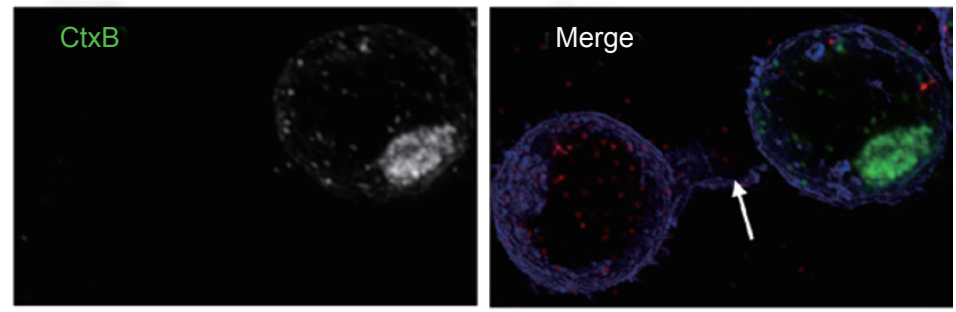

D $\mathrm{CH}-11$ or FasL $30 \mathrm{~min}$

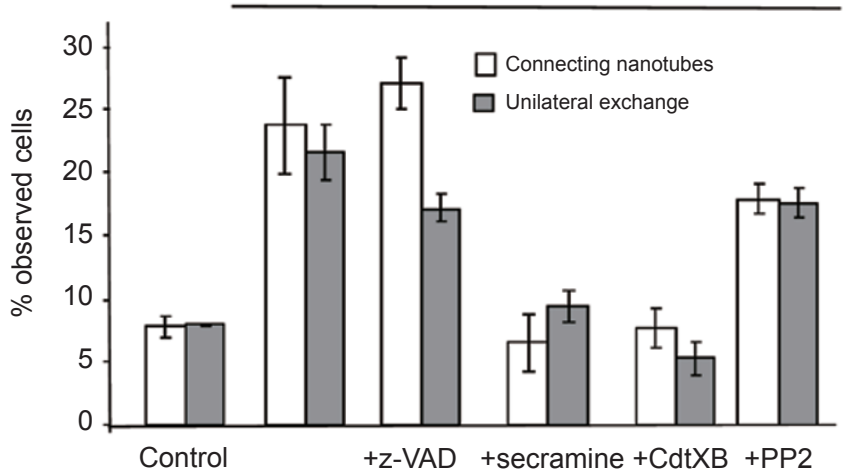

Figure 3 Fas-enhanced nanotubes facilitate intercellular membrane transfer. (A) Connecting nanotubes were observed in live Jurkat cells using bright field images $(\times 100$ objective). Every nanotube formed was cumulatively counted, even if transient (average nanotube duration was 15.4 min after Fas stimulation, cf. Supplementary information, Movie S1). Cells were treated with FasL or $\mathrm{CH}-116$ min before data acquisition. (B) Connecting membrane nanotubes transiently increased after Fas activation with either FasL or $\mathrm{CH}-11$ for $30 \mathrm{~min}$. Data represent averages \pm S.D. with Fas stimulation before (control, $n=5$ experiments) or after pre-treatment with $0.1 \mu \mathrm{g} / \mathrm{ml} \mathrm{CdTXB}$ or $20 \mu \mathrm{M}$ secramine A for $30 \mathrm{~min}$ ( $n=3$ experiments), which allowed full cell permeation and Rho GTPase inhibition [13]. Differences were highly significant after 20 min (e.g., $P<0.02$, MannWhitney). (C) Jurkat cells were treated with $50 \mu \mathrm{M}$ z-VAD for $30 \mathrm{~min}$ before being separately stained with a dual PE-Cy5 conjugate of anti-CD59 (1:15) or Alexa488-conjugated CtxB (green), and then mixed in the presence of $0.5 \mu \mathrm{g} / \mathrm{ml}$ of $\mathrm{CH}-11$ for $30 \mathrm{~min}$ (including attachment on cover slips). After fixation, cells were permeabilized with $0.1 \%$ saponin and then stained with Alexa Fluor 350-conjugated HPA (blue). Deconvolution microscopy (×60 objective) was undertaken with a PE excitation filter and a Cy5 emission filter (coloured in red) to minimize bleeding with the green channel. Of note, anti-CD59 antibody and CtxB conjugates were not activating their target molecules on the cell surface [13]. Moreover, CtxB was used as in the studies of clathrin-independent endocytosis [13] rather than a reporter for lipid rafts [2, 39]. Arrowheads point to CD59-labelled elements uni-laterally migrating along the connecting nanotube (large arrow) and then inserted in the CtxB-stained cell. (D) Pharmacological modulation of connecting membrane nanotubes and uni-lateral exchange of CD59 was evaluated after Fas activation for $30 \mathrm{~min}$ as in $\mathbf{C}$. Cells were pre-treated with secramine and CdTXB as in the experiment of part B, while z-VAD and PP2 were added at 50 and $10 \mu \mathrm{M}$, respectively, and incubated for 30 min before $\mathrm{CH}-11$ treatment. Control cells were treated with a non-activating anti-Fas mAb. Histograms represent the average \pm S.D. of $n=4$ with inhibitors and $n=6$ experiments without inhibitors. Differences were statistically significant for secramine and CdTXB only $(P<0.03$, Mann-Whitney). 
(CdTXB) [16], as well as the CDC42-specific inhibitor secramine A [17] were particularly effective in blocking Fas-induced nanotube formation (Figure 3 and Supplementary information, Figure S3C).

\section{Fas-enhanced nanotubes facilitate Rho GTPase-depen- dent exchanges}

Nanotubular connections have the capacity of transferring membrane elements among connected cells $[8,15]$. This transfer can be either unidirectional (or uni-lateral), when a 'donor' cell produces a nanotubular structure along which it sends membrane elements to an 'acceptor' cell, or bidirectional (bi-lateral), when two or more cells mutually exchange material via connecting protrusions. First, we evaluated the unidirectional transfer of membranes carrying CD59 from cells labelled with antibodies against CD59 to cells stained with other markers (Figure 3C, cf. [14]). The transfer of CD59 evaluated in this way increased fourfold $30 \mathrm{~min}$ after Fas stimulation (Figure 3D). Membranes carrying anti-CD59 antibodies were present in over half of the nanotubes connecting a CD59labelled cell with another cell stained differently (Figure $3 \mathrm{C})$. However, these membranes were virtually absent in the few nanotubes observed in cells pre-treated with Rho GTPase inhibitors, which also showed a reduced exchange of the same membranes (Figure 3D). In contrast, the Fas-enhanced exchange was unaffected by caspase inhibition (Figure 3D).

Besides nanotubes, intercellular exchange of membranes can also occur through the process of trogocytosis [9, 18-20]. Trogocytosis, besides being sensitive to agents that perturb the actin cytoskeleton, is additionally inhibited by PP2 [19, 20], a broad tyrosine kinase inhibitor, which has not been tested on membrane nanotubes [15]. We thus investigated whether treatment with PP2 affected the exchange promoted by Fas stimulation. The results showed that PP2 induced no significant change in membrane nanotubes or uni-lateral transfer of CD59 (Figure 3C and Supplementary information, Figure S3C). Hence, connection via nanotubes, rather than trogocytosis, is the major mechanism of Fas-enhanced uni-lateral exchange of membranes.

\section{Fas activation stimulates bi-lateral transfer of cellular components via nanotubes}

Recently, it has been reported that membrane nanotubes in Jurkat and other $\mathrm{T}$ cells can promote the spread of virus particles but not of cytoplasmic components [14]. These nanotubes appeared to differ from open tunneling connections that had been previously characterized in other cells $[8-10,15]$. Diverse nanotubular connections appeared to be present in Fas-stimulated cells as indi- cated by high-resolution images with fluorescent markers and transmission electron microscopy (Figures 2-4 and Supplementary information, Figure S2).

Because morphological evidence would be insufficient to define different types of nanotubular connections [15, 21], we next investigated the functional capacity of membrane nanotubes to also drive the bidirectional exchange of membranes between connected cells. This would be consistent with open endings $[8,15,21]$, since seamless nanotubes facilitate only unidirectional transfer of membranes [14]. To evaluate the bidirectional exchange of membranes, we measured the frequency of nanotubes that connected cells stained with two different membrane markers and simultaneously contained membrane elements labelled with each of these markers.

We concentrated first on experiments where Jurkat cells were separately stained with green fluorescent antiCD59 and infrared-fluorescent anti-CD81, and then mixed together in the presence of the Fas agonist $\mathrm{CH}-11$ (Figure 4A). Each antibody alone exhibited a comparable frequency of occurrence within membrane nanotubes (Figure 4B). Control cells showed a similar frequency of empty nanotubes, i.e., those that carried no marker at all (white histograms in Figure 4C), and also a negligible number of nanotubes containing both markers (black histograms in Figure 4C). In contrast, 30 min treatment with $\mathrm{CH}-11$ induced a 20 -fold increase in the number of nanotubes carrying both CD59 and CD81 (Figure 4C). Pre-treatment with z-VAD or PP2 did not reduce the frequency of these double stained nanotubes, whereas pretreatment with CdTXB strongly reduced their number (Figure 4C). By extending these studies, we found that the level of connecting nanotubes carrying two membrane markers was directly proportional to the lowest frequency of nanotubes carrying a single marker. For instance, a mixture of CD59-stained cells with cells stained green with $\mathrm{CtxB}$ (cf. Figure 3C) showed $23.3 \%$ of connecting nanotubes that contained both CD59 and CtxB, reflecting the lower frequency of nanotubes containing only CtxB (32.6\%) than those containing only CD59 (62.8\%). However, nanotubes did not carry both CD59 and $\mathrm{CtxB}$ in the presence of either secramine or CdTXB. Hence, inhibition of Rho GTPases eliminated the concomitant presence of differently stained membranes in connecting nanotubes, consequently abolishing bidirectional transfers of membranes mediated by nanotubes.

Open-ended (tunneling) nanotubes can also mediate the transfer of cytosolic components, contrary to trogocytosis $[15,19,21]$. We thus investigated whether Fas stimulation led to an exchange of cytosolic material along membrane nanotubes. Adherent (293T) cells transfected with cytosolic yellow fluorescent protein (YFP) 
Bi-directional exchange of membranes via nanotubes

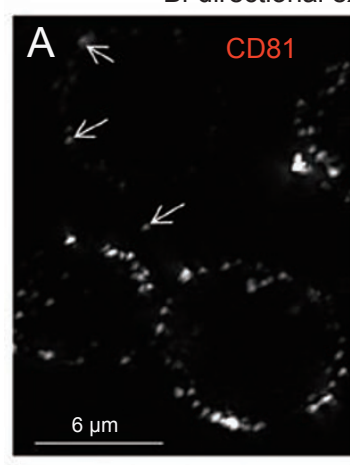

B

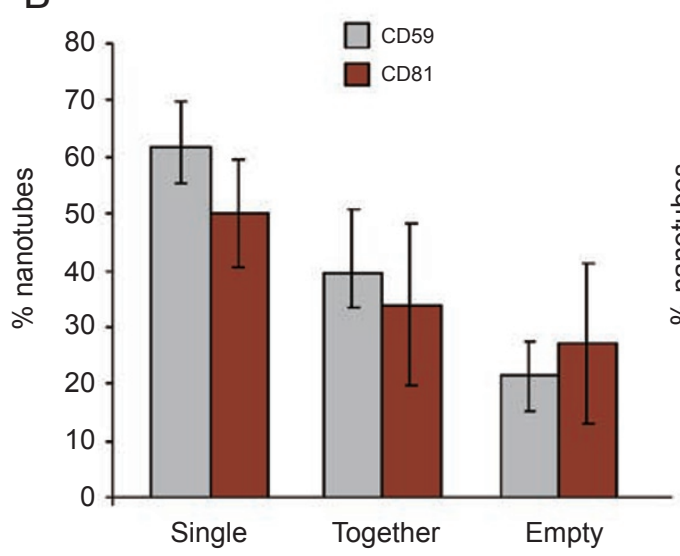

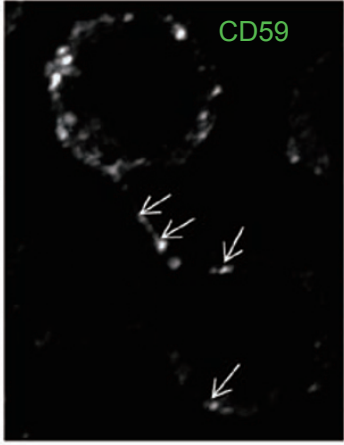

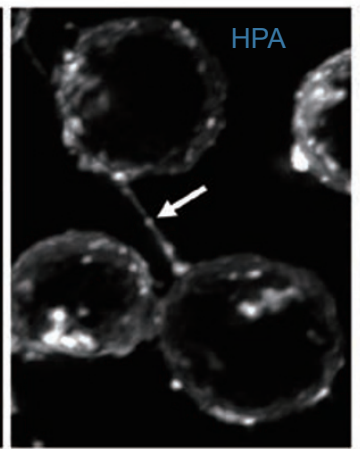

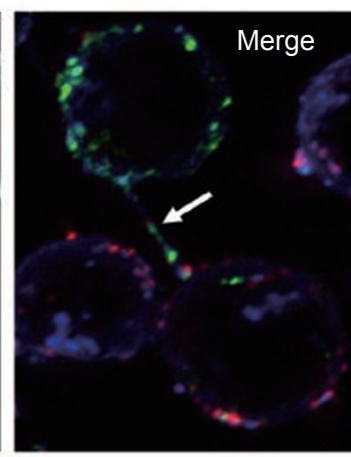

$\mathrm{CH}-1130 \mathrm{~min}$
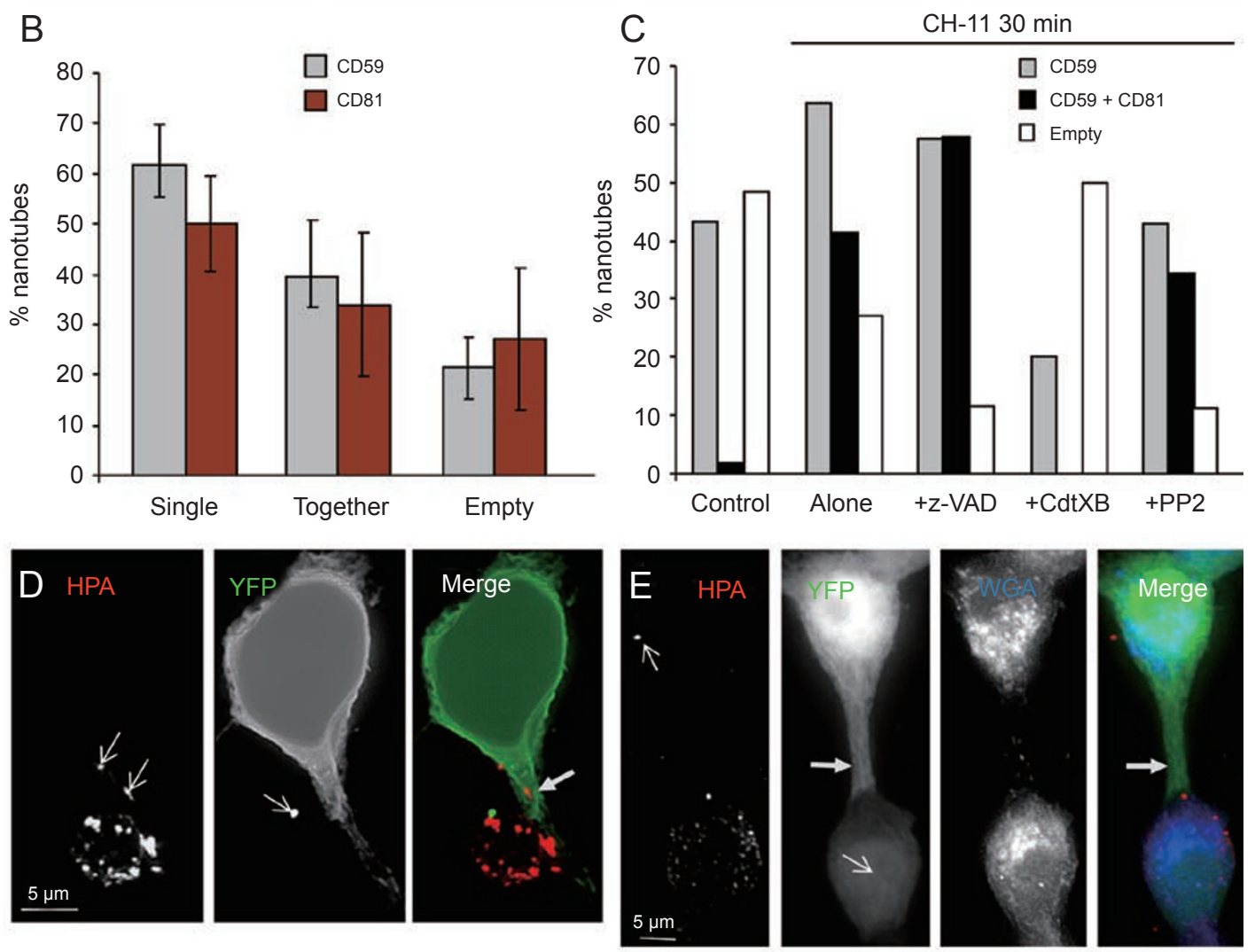

Figure 4 Fas signalling enhances bidirectional exchanges along nanotubes. (A) An aliquot of Jurkat cells were pre-stained green with anti-CD59 and then mixed with another aliquot that had been stained with allophycocyanin (APC)-conjugated anti-CD81 in the presence of $\mathrm{CH}-11$ for $30 \mathrm{~min}$, including attachment on cover slips. After fixation, cells were stained with blue HPA and imaged as in the experiment of Figure 3C. Note the connecting membrane nanotube (arrow) that carries both CD59-labelled membranes and CD81-labelled membranes (arrowheads). (B) The frequency of occurrence of nanotubes containing only CD59 or CD81 staining was computed for $n=4$ separate experiments like that in A. No significant difference was found with various tests. (C) Pharmacological profile of the bidirectional exchange of CD59- and CD81-labelled membranes was evaluated by counting connecting nanotubes that contained either CD59 alone (grey) or both CD59 and CD81 (black). Nanotubes that did not contain any labelled particle after background subtraction were scored as empty (white). Histograms represent the average of two separate experiments with approx. 240 nanotubes analysed. Inhibitors were pre-incubated as in the experiments of Figure 3. (D) A membrane nanotube connects a YFP-expressing 293T cell (top) with a Jurkat cell labelled with red HPA (bottom). Labelled Jurkat cells in RB buffer were layered over cover slips with attached $293 \mathrm{~T}$ cells $24 \mathrm{~h}$ after YFP transfection and incubated for $30 \mathrm{~min}$ in the presence of $0.5 \mu \mathrm{g} / \mathrm{ml} \mathrm{CH}-11$ before fixation. Note the red-labelled elements (arrowheads in the left panel) that migrate along the nanotube (arrow) and the bright green element (arrowhead in central panel) that is associated with the red cell. (E) A large membrane nanotube connects a YFP-transfected cell with a Jurkat cell (bottom) in the same settings as in D, but with a clear diffusion of cytosolic YFP into the red cell (arrowhead). A red-labelled membrane element is also present in the 293T cell (arrowhead in left panel). 

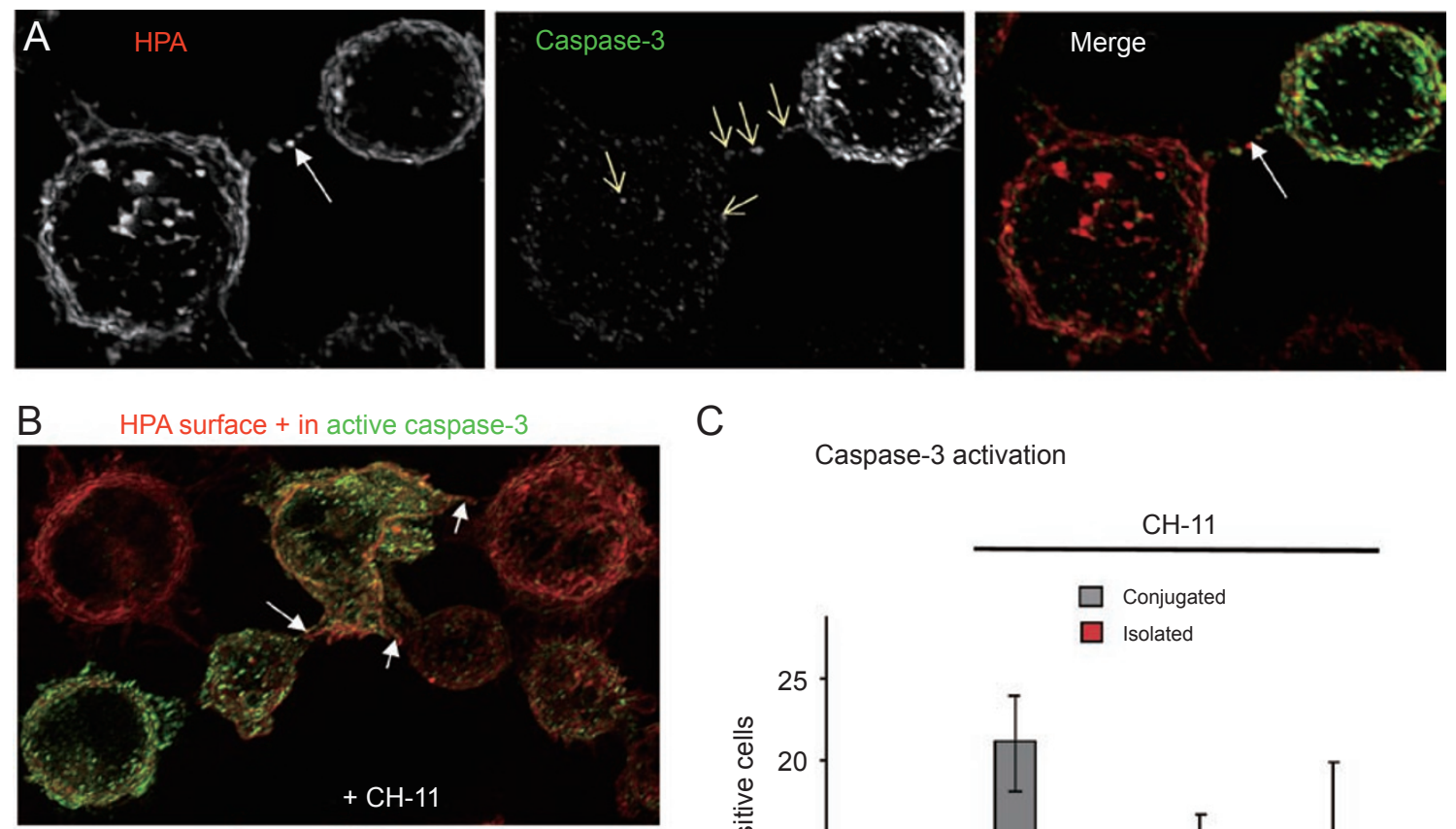

C

Caspase-3 activation
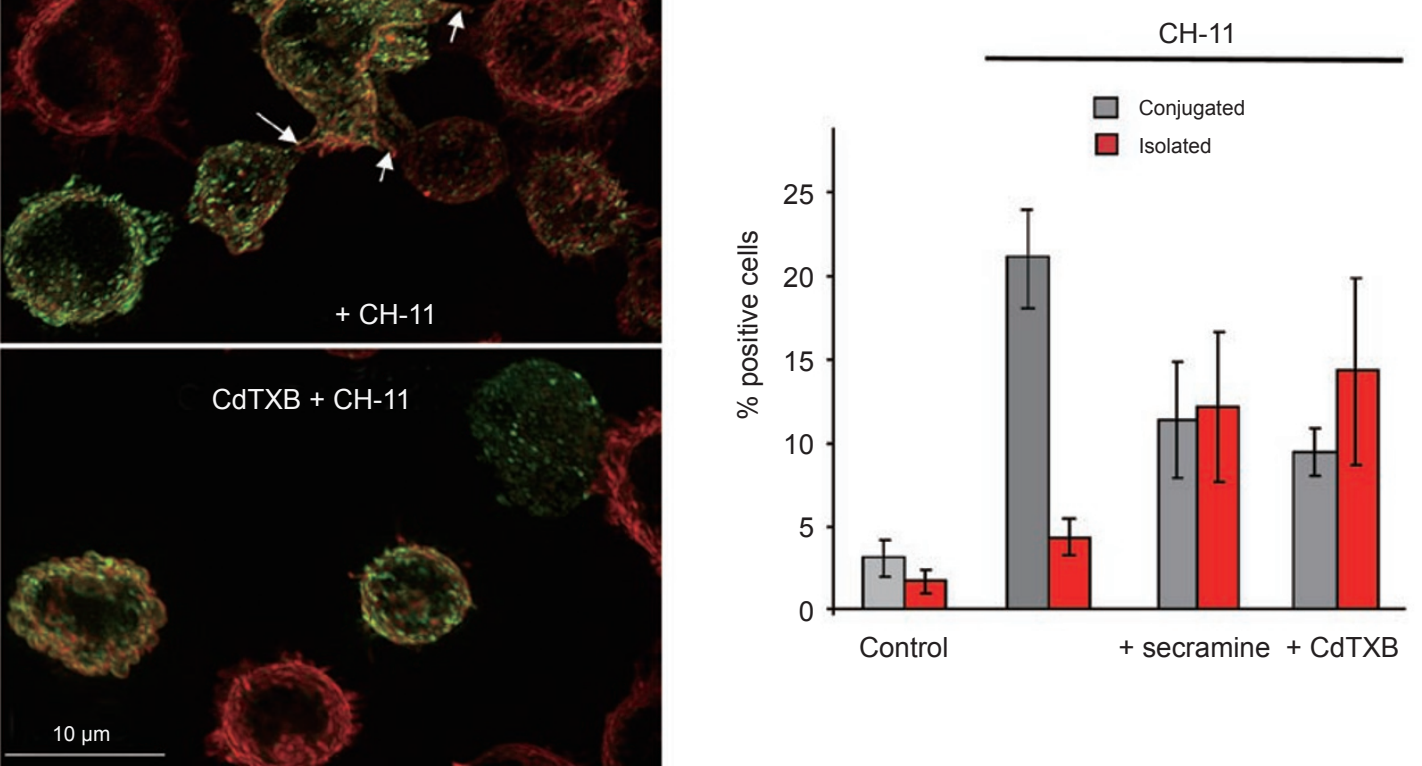

$$
\text { (n) }
$$

Figure 5 Membrane nanotubes transfer active caspases and contribute to death propagation. (A) One-half of a Jurkat suspension was treated with $\mathrm{CH}-11$ for $30 \mathrm{~min}$, to activate caspases in advance, then washed and mixed to the other half that was left untreated. After 15-min attachment on cover slips, cells were fixed, permeabilized, stained for active caspase-3 and surface decorated with red HPA as in the experiment of Figure 1B. Note the nanotube (arrow) connecting a Fas-activated cell (top) and containing elements positive for active caspase- 3 that migrate into the bottom cell (thin arrows). (B) The experiment was conducted as in A, except that the aliquot of untreated cells was allowed to endocytose red HPA before mixing and attachment. Note the connecting nanotubes containing active caspase-3 (arrows) between asymmetrically stained cells. The images show representative $\times 60$ fields of Jurkat cells following 40 min incubation with $\mathrm{CH}-11$ (cf. Figure 1B). (C) Histograms represent the averages \pm S.D. of the positive scores for active caspase- 3 obtained from $n=4$ separate experiments as in $\mathbf{D}$ plus one performed with Imagen-Biotech platform (cf. Supplementary information, Figure S1B). All results were significantly higher than the values of control cells (treated with a non-activating anti-Fas antibody). However, samples with Rho GTPase inhibitors showed very different values, for both isolated and conjugated cells, from those with $\mathrm{CH}-11$ alone $(P=0.02$ for conjugated cells plus CdTXB and $P=0.03$ for cells with secramine, Mann-Whitney). Nearly 8000 cells were examined.

(cf. [21]) showed an increased number of nanotubular protrusions and exchanged YFP with Jurkat cells labelled with red membrane markers (Figure 4 and Supplementary information, Figure S3D). Connecting nanotubes appeared to carry HPA-labelled vesicles (Figure 4D and 4E and Supplementary information, Figure S3D) while being filled with cytosolic YFP, which could be observed to spread into the cytosol of conjugated cells (Figure
4E and Supplementary information, Figure S3D). Cotransfection with cyan fluorescent protein (CFP) fused to a nuclear protein indicated that only YFP, predominantly located in the cytosol, could be effectively transferred along nanotubes. In diverse cell conjugates, bright green membrane elements associated with red-labelled cells (Figure 4D), indicating the additional exchange of cytosol-containing vesicles like exosomes. However, these 
green elements were not present in non-conjugated cells.

Membrane nanotubes allow the transfer of active caspases between connecting cells

The implication that emerged from the above studies was that membrane organelles and cytosolic elements could diffuse between Fas-stimulated cells predominantly via open-ended nanotubes. Could nanotubes also mediate exchange of activated caspases, thereby contributing to the propagation of Fas-induced apoptosis? To
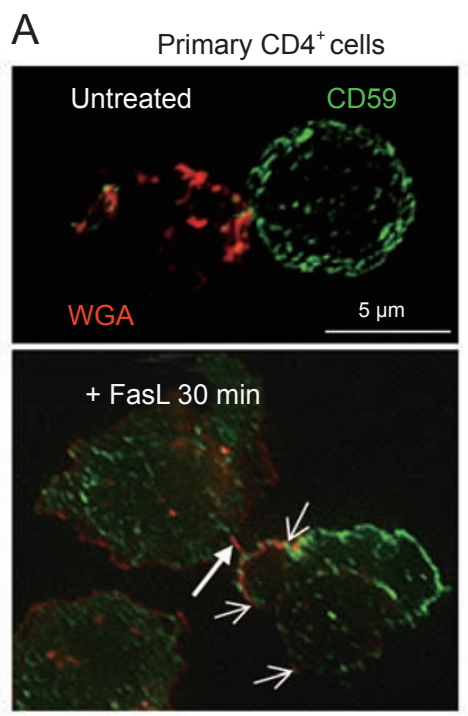

B

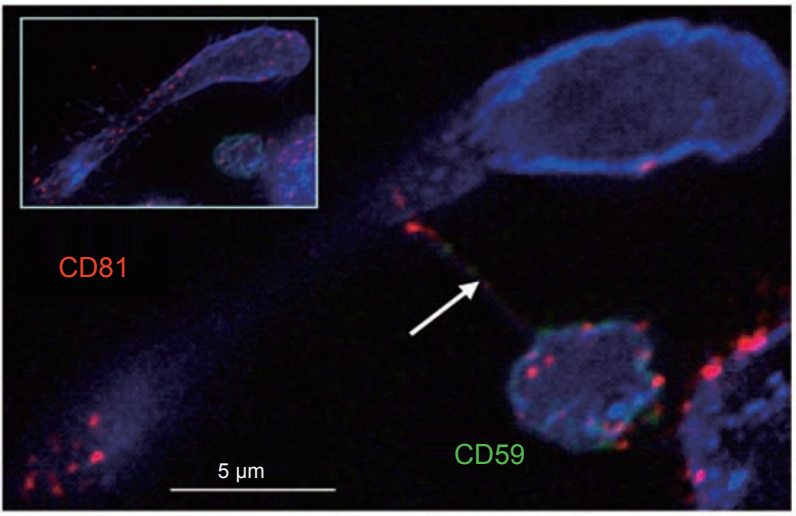

C Exchange of cytoplasm
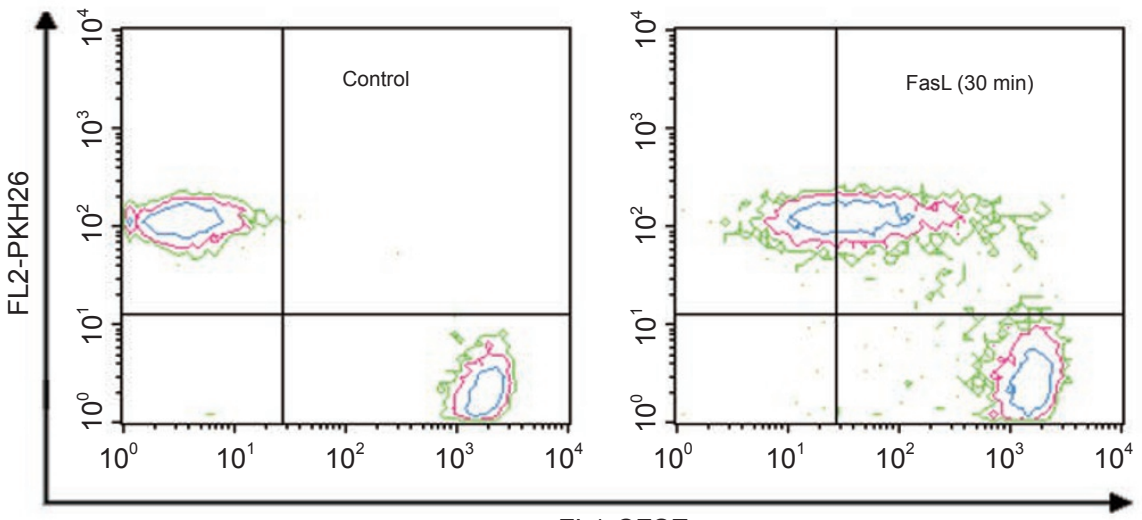

FL1-CFSE

D

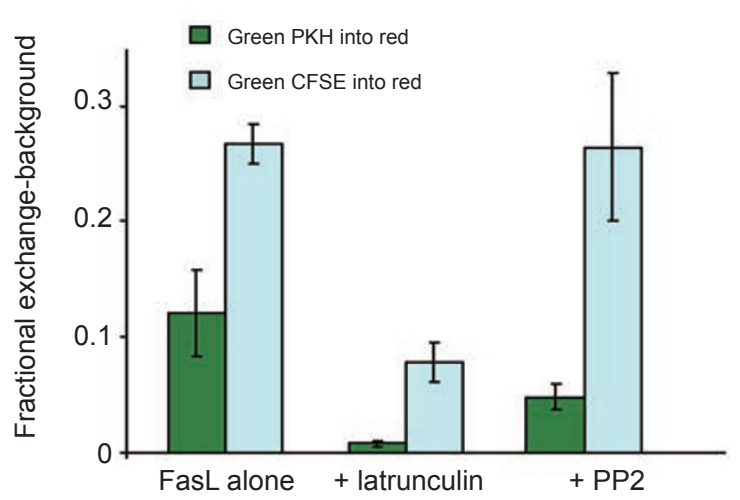

Figure 6 Fas signalling enhances membrane and cytosol exchange in primary CD4 ${ }^{+} \mathrm{T}$ cells. (A) CD4 ${ }^{+} \mathrm{T}$ cells, activated with PHA, were separately stained with $5 \mu \mathrm{g} / \mathrm{ml}$ TRITC-WGA (red) or FITC-conjugated anti-CD59 (green, incubated at 1:10), washed and then mixed as in the experiment of Figure 3C (but with FasL instead of $\mathrm{CH}-11$ ). Representative couplings of FasL-treated cells (bottom panel) show increased exchange of red-labelled membranes (arrow heads) and also a connecting nanotube (thick arrow). (B) CD4 ${ }^{+} \mathrm{T}$ cells, activated with ionomycin and PMA, were separately stained with green anti-CD59 and infrared anti-CD81, and then treated as in the experiment of Figure 4A, except that AlexaFluor350-conjugated WGA was used for surface decoration (blue). The main image is the projection of 12 central $z$ sections showing a thin connecting nanotube (arrow) that contains both CD81 (red) and CD59 (green) membrane elements in transit between cells. The insert shows the projection of the full set of 24 sections for the same $\times 60$ image. (C) Activated $C D 4^{+}$cells $T$ cells were separately stained red with PKH 26 and green with $0.5 \mu \mathrm{M}$ CFSE, a cytosolic marker [20]. In the dot plot obtained after filtering out cell conjugates [30], cells stained with PKH26 and CFSE were completely separated from each other in the control (left) but showed a population of double positive after $30 \mathrm{~min}$ of FasL treatment (right). Data is representative of four separate experiments. (D) Quantitative evaluation of the exchange of cytosolic (CFSE, light blue histograms) and membrane (PKH67, dark green histograms) elements was conducted in $n=4$ experiments like that in $\mathrm{C}$ but using the method of Poupot and Fournie [22]. The decrease in the average exchange of both markers that was observed after pre-treatment with PP2 was not significant, while that observed after pre-treatment with latrunculin was significant at $P=0.03$ (Mann-Whitney). 

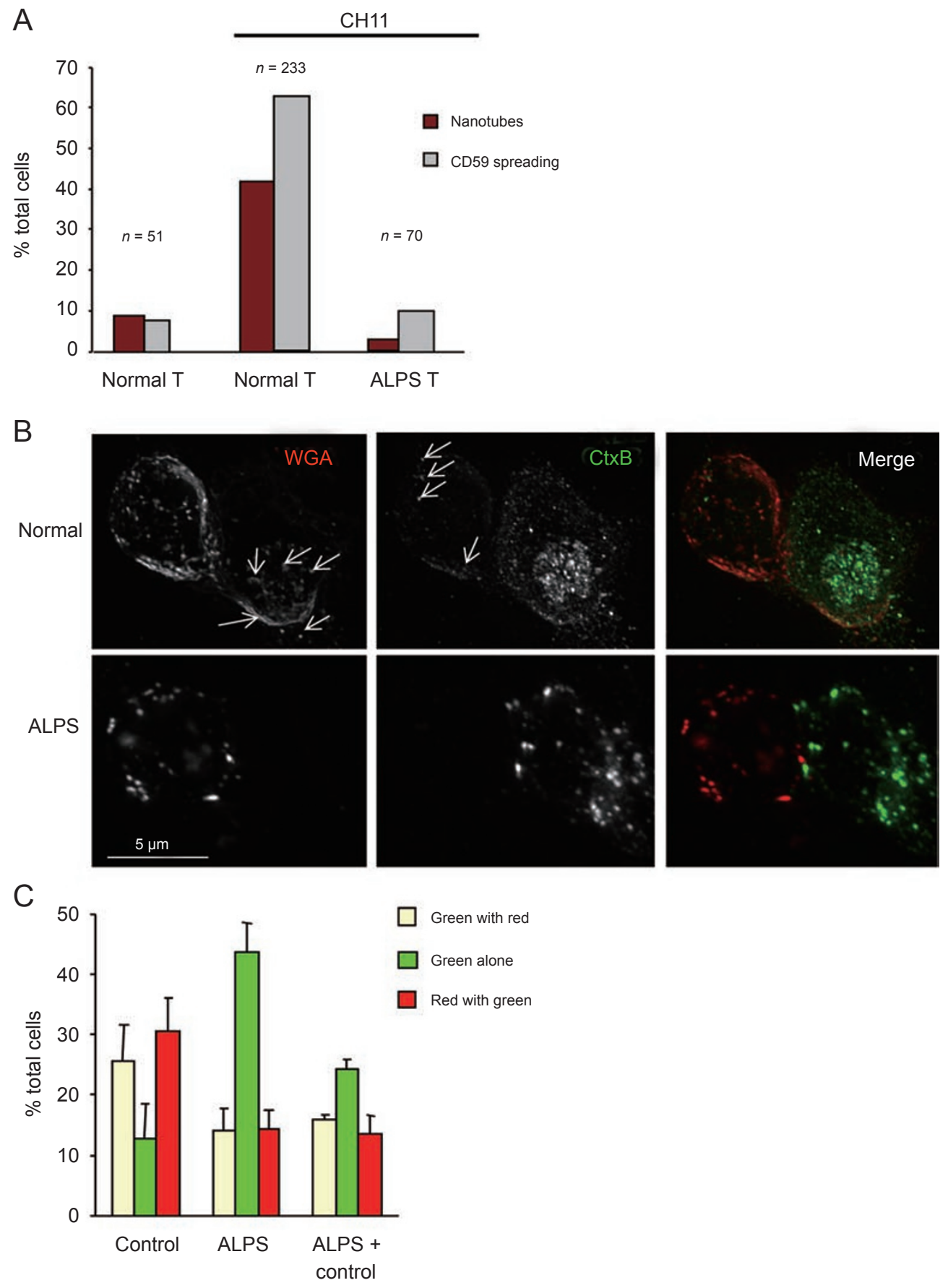

Figure $7 \mathrm{~T}$ cells from ALPS patients are defective in membrane nanotubes and transfer. (A) Quantitative evaluation of connecting nanotubes and CD59 spreading, a reporter of Fas-enhanced endocytosis [13], was conducted in two equivalent experiments using activated $\mathrm{CD}^{+} \mathrm{T}$ lymphocytes as in the experiment of Figure 6B; the histograms represent the average values obtained after analysing the indicated number of cells. (B) Activated $\mathrm{CD} 4^{+} \mathrm{T}$ cells from healthy donors (normal) and the ALPS patient (bottom panels) were separately stained with red WGA and green CtxB, and then mixed in the presence of FasL for 30 min before fixing and imaging with deconvolution microscopy $(\times 100$ objective). Arrows indicate membrane elements that exchange between the conjugated cells. (C) Data show the cumulative analysis of $n=5$ experiments like that in B by scoring $>100$ cells for each sample from three ALPS patients (besides the one used in parts A-C, a second harbouring the homozygote deletion c475-488 and a third with the mosaic mutation c677-1G>T within the Fas gene). Note that the maximal score for each type of cells would be $50 \%$. Differences between ALPS and normal cells were significant: $P<0.05$ for 'green with red' or 'red with green' and $P<0.02$ for 'green alone' (Mann-Whitney). 
answer this question, we undertook immunofluorescence analysis of active caspase- 3 in experiments in which half of cells were treated with $\mathrm{CH}-11$ and then mixed with untreated cells. Membrane nanotubes that connected a cell with strong caspase- 3 activation to another which was originally untreated with $\mathrm{CH}-11$ appeared to contain elements carrying active caspase- 3 that moved along the connection and then diffused within the target cell (Figure $5 \mathrm{~A}$ ). However, the interpretation of these observations was not straightforward, because it was almost impossible to establish whether a positive caspase-3 staining originated within a target cell or really came from a donor cell showing a more advanced stage of caspase activation.

We then resorted to the quantitative analysis previously used for the evaluation of bi-lateral membrane transfer (cf. Figure 4B) and measured the frequency of nanotubes carrying caspase- 3 . We found that $71.5 \%$ of nanotubes connecting a cell showing high caspase-3 activation with another cell having negligible caspase- 3 activation (Figure 5B) contained caspase-3-positive elements after Fas stimulation. This frequency decreased to $19.5 \%$ in samples treated with either CdTXB or secramine before Fas activation (this result was obtained by evaluating an equivalent number ( $\mathrm{ca}$. 50) of connecting nanotubes, which implied the examination of a larger set of microscopic fields, due to the reduced frequency of nanotubes after blocking Rho GTPases). Since the same inhibitors of Rho GTPases did not significantly change the level of caspase- 3 activation within cells (Degli Esposti, unpublished observations and ref. [13]), the large decrease in the frequency of connecting nanotubes that contained active caspase- 3 indicated a reduced intercellular transfer of the caspase via the same nanotubes.

To verify whether this reduced transfer affected the spread of death within $T$ cell populations, we next considered whether Rho GTPase inhibitors could alter the previously observed asymmetric rates of apoptosis (Figure 1). Indeed, the asymmetric distribution of caspase-3 activation, which was fourfold higher in conjugated or nanotube-connected cells than isolated cells, disappeared in the presence of Rho GTPase inhibitors (Figure 5C). This result also suggested that the uneven distribution of death indicators (Figures 1 and 5) did not derive from the inherently stochastic nature of apoptosis but reflected spreading of death signals between cells.

Membrane nanotubes and exchange of cytoplasmic components in primary T cells

Membrane nanotubes carrying two different markers were also seen in primary $\mathrm{CD} 4^{+}$cells that had been activated and then treated with FasL (Figure 6A and 6B).
Primary activated $\mathrm{T}$ cells showed hardly any membrane nanotubes and low levels of membrane exchange before Fas stimulation (Supplementary information, Figure S4). Within 60 min of Fas stimulation with FasL, a fivefold increase in the exchange of membrane markers like CD59 was detected (Figure 6A and 6B), a result that was confirmed by flow cytometry (Supplementary information, Figure S4A). While CtxB and CD59 showed basal and Fas-enhanced levels of transfer that were comparable in Jurkat and primary $\mathrm{T}$ cells, lipophilic stains routinely used for measuring membrane exchanges in lymphocytes, PKH26 in particular [20,22], exhibited much reduced exchanges (Supplementary information, Figure S4B, S4C).

We next applied flow cytometry to evaluate whether Fas stimulation led to an exchange of cytosolic components also in primary $\mathrm{T}$ cells. Using the cytosolic stain carboxyfluorescein succinimidyl ester (CFSE) [20], we found that Fas stimulation induced a large exchange of green fluorescence in cells stained red with either PKH26 or the mitochondrial marker TMRE (Figure 6C and 6D). These cytosolic exchanges were larger and less affected by PP2 than those detected with the green lipophilic marker PKH67 (Figure 6C and 6D). However, all exchanges were blocked by actin-disrupting latrunculin (Figure 6D). Consequently, our flow cytometry results indicated that Fas stimulation increased the exchange of cytosolic as well as membrane elements between T cells, consistent with transfer via nanotubes but not trogocytosis.

Fas-enhanced membrane nanotubes are not present in $T$ cells from ALPS patients

Next, we investigated whether nanotube formation and membrane transfer were altered in $\mathrm{T}$ lymphocytes from ALPS patients harbouring pathological mutations in Fas signalling (type Ia ALPS $[6,7]$ ). We reasoned that if membrane nanotubes were an important form of intercellular communication for Fas-stimulated $\mathrm{T}$ cells, they might not be present in pathological conditions resulting from primary Fas defects leading to reduced clearance of activated T cells, as in ALPS patients. Indeed, membrane nanotubes were not found to connect activated $\mathrm{T}$ cells from ALPS Ia patients (Figure 7A and Supplementary information, Figure S4D).

Activated T cells from Type Ia ALPS also exhibited markedly reduced membrane exchanges. We studied $\mathrm{T}$ cells from a patient with type 1 ALPS due to a 4-bp duplication in exon 9 creating a premature stop codon (216), who from 18 months of age had developed characteristic paracortical expansion with $44 \% \mathrm{CD}^{+} \mathrm{CD}^{-} \mathrm{CD}^{-} 8^{-} \mathrm{T}$ cells (normally $<1 \%$ ). Caspase activation and exchange of membrane markers following FasL treatment was 

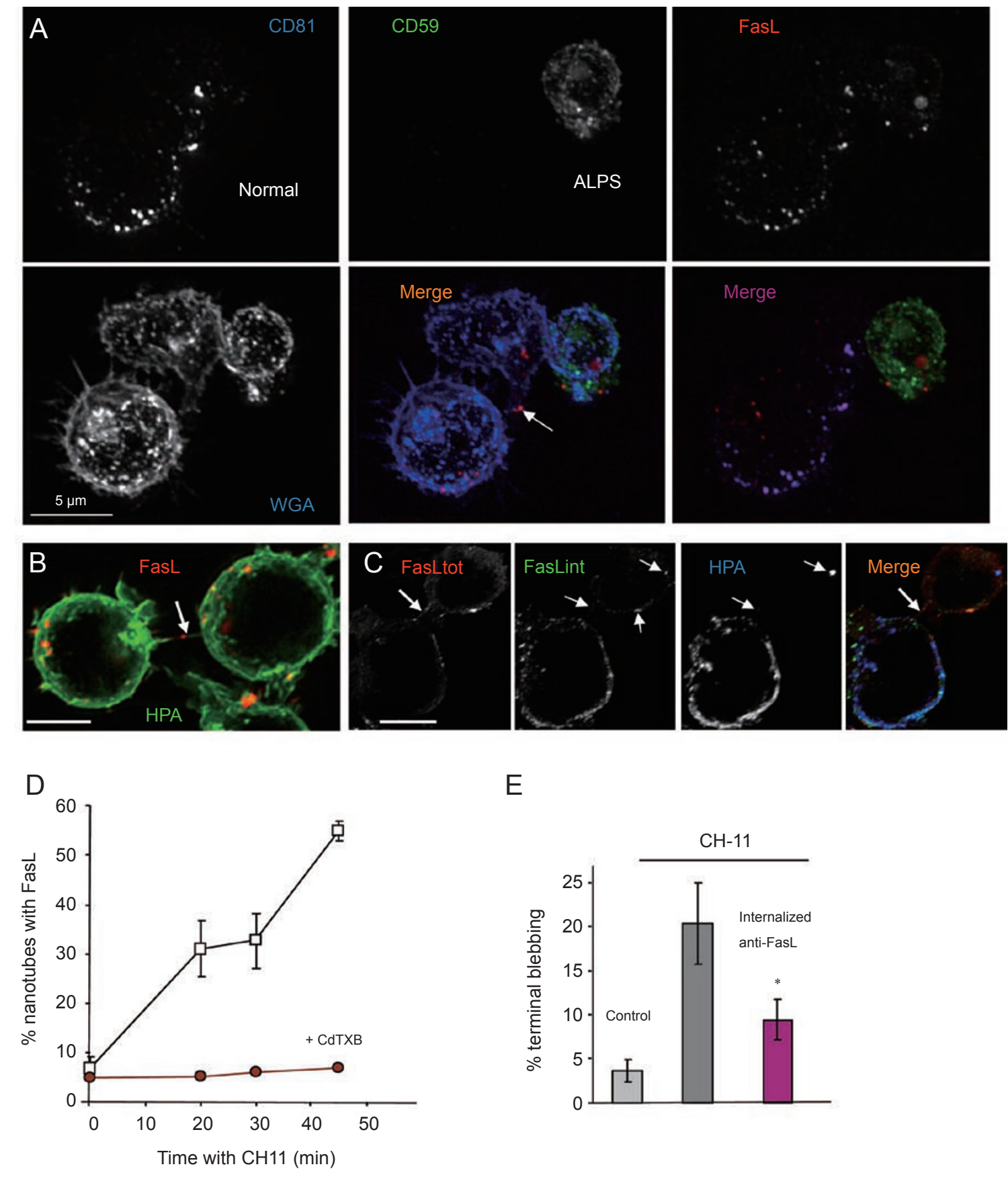

$E$

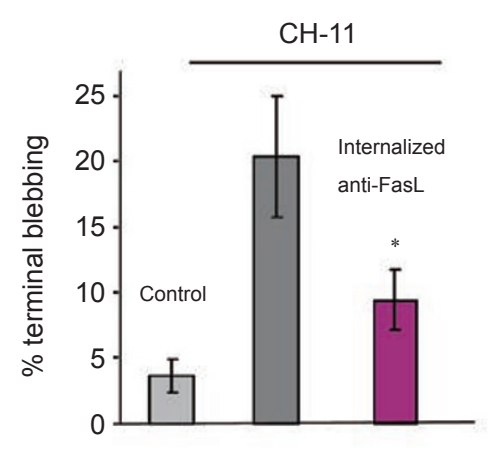

Figure 8 Nanotubes transfer FasL among T cells. (A) Primary CD4 ${ }^{+}$cells from a normal individual and the same ALPS patient used in the experiment of Figure 7A were activated with ionomycin and PHA, and then IL-2. After washing and suspension in RB buffer, normal T cells were incubated with APC-conjugated anti-CD81, while those from the ALPS patient were incubated with FITC-conjugated anti-CD59 for 20 min to induce effective internalization of the antibodies. Subsequent to washing, normal and ALPS cells were mixed in a 2:1 ratio and treated with $\mathrm{CH}-11$. After fixation and permeabilization with saponin, cells were labelled with PE-conjugated NOK-1 (FasL) and decorated with WGA (blue). Deconvolved images were then taken with a $\times 100$ objective and split in the indicated colours for analysis. Note that no CD81-positive or CD59-positive element was exchanged between the normal cell conjugated to the ALPS cell, while a connecting nanotube (arrow) transferred FasLpositive elements between normal cells. FasL staining in the ALPS cell remained clustered, contrary to the dispersed distribution in normal cells. (B) Jurkat cells were treated with $\mathrm{CH}-11$ for 20 min and then immunostained with PE-conjugated NOK-1 (red). After counterstaining the cell surface with green HPA, cells were imaged with a $\times 100$ objective. FasL is present within the connecting nanotube (arrow). (C) Enhanced internalization of NOK-1 was conducted together with blue HPA as a tracer. Washed blue cells were then mixed with unlabeled cells in the presence of $\mathrm{CH}-11$ for $15 \mathrm{~min}$. After fixation and permeabiliza- 
severely impaired in the patient's T cells (Figure 7B and 7C). For instance, while FasL treatment of control T cells led to exchange of red fluorescent WGA into cells initially displaying green $\mathrm{CtxB}$ only, conjugated $\mathrm{T}$ cells from the ALPS patient showed little difference before and after FasL treatment (Figure 7B, lower panels). Equivalent results were obtained by analysing the exchange of membranes in T cells from other ALPS patients harbouring different Fas mutations (Figure 7C), suggesting that nanotube formation is defective in this condition.

Fas activation stimulates the transfer of endogenous FasL along nanotubes

Given that FasL appears to be crucial in peripheral deletion of T cells, which is impaired in ALPS patients [1, $2,7]$, we investigated whether endogenous, membranebound FasL could be exchanged along nanotubes connecting Fas-stimulated T cells. By using the anti-FasL antibody NOK-1 (the specificity of which was thoroughly verified in our settings), we found that an enhanced number of connecting nanotubes contained FasL-enriched membrane elements after Fas stimulation of primary and Jurkat $\mathrm{T}$ cells (Figure 8). However, membrane nanotubes containing FasL were hardly observed in $\mathrm{T}$ cells from ALPS patients, even when these cells were conjugated to normal $\mathrm{T}$ cells exchanging membranes with each other (Figure 8A). Of note, morphological signs of Fasinduced death were absent in ALPS T cells, while normal $\mathrm{T}$ cells displayed ca. $8 \%$ blebbing in experiments like that of Figure 8A (cf. [13]).

To verify whether endogenous FasL was exchanged among conjugated cells, we took the advantage of our observation that short treatment with $\mathrm{CH}-11$ increased the uptake and subsequent internalization of anti-FasL antibodies (Figure 8C and Supplementary information, Figure S4E), which normally cannot access endogenous FasL because its traffic is restricted within secretory lysosomes [23]. T cells that had internalized anti-FasL antibodies then transferred them to unlabelled cells, pre- dominantly using connecting nanotubes (Figure 8C and Supplementary information, FigureS4E), since they did not transfer the same antibodies after CdTXB treatment. In one representative experiment, CdTXB reduced the exchange of internalized NOK-1 from 18.2 to $5.4 \%$ after 45 min of Fas stimulation. Indeed, inhibition of Rho GTPases eliminated FasL from connecting nanotubes (Figure $8 \mathrm{D}$ and data not shown).

Was the nanotube-mediated transfer of FasL responsible for an early wave of cell-assisted death? To address the question, we exploited the fact that the anti-FasL antibodies used here also blocked the function of FasL, since they prevent FasL interaction with its cognate receptor [24]. Consequently, endogenous FasL bound to blocking antibodies would not contribute to the spread of cell death, if this death were dependent upon Fas interaction with exchanged molecules of FasL. Confirming this prediction, cells with internalized anti-FasL antibodies showed a twofold decrease in blebbing when compared with cells not exposed to the same antibodies (Figure $8 \mathrm{E})$. Data with live cell imaging matched the results obtained with immunocytofluorescence, further sustaining the concept that early transfer of FasL contributed to intercellular spreading of death.

\section{Discussion}

In this study, we show for the first time that activation of death receptors induces the extensive formation of interconnecting membrane nanotubes, through which membrane elements move from cell to cell. After initiation of Fas signalling, T lymphocytes appear to increasingly communicate via these nanotubes, but not when they harbour mutant Fas receptors as in those from ALPS I patients (Figures 7 and $8 \mathrm{~A}$ ). Hence, a functional Fas is required to initiate intercellular communication. Intriguingly, TRAIL increases the formation of connecting nanotubes similarly to FasL (Supplementary information, Figure S3), suggesting that their cognate death recep-

tion, the total complement of endogenous FasL was stained with a goat antibody followed by Cy3-conjugated anti-goat (red). Conversely, the previously internalized NOK-1 was stained with FITC-labelled anti-mouse (green). Both NOK-1 and blue HPA were found into connected cells exhibiting predominant red staining for non-internalized FasL. Deconvolved images were obtained with a $\times 100$ objective. The thick arrow indicates a connecting nanotube containing FasL and arrowheads indicate elements exhibiting co-localized staining with anti-FasL antibodies. (D) Membrane nanotubes containing FasL increased during Fas stimulation, but not in the presence of CdTXB (circles, averages of two experiments). Data without the inhibitor (squares) were the averages of $n=6$ experiments. Approximately, 500 nanotubes were evaluated. (E) Internalized anti-FasL antibodies reduce the extent of blebbing after $40 \mathrm{~min}$ of cumulative treatment with $\mathrm{CH}-11$. Cells not exposed to anti-Fas $\mathrm{L}$ antibodies exhibited more blebbing than cells with internalized antibodies $(P=0.012$, Mann-Whitney, and $P=0.002$, ANOVA). Data was obtained from four to six separate experiments after scoring a total of 1560 cells. Results of a parallel experiment with live cells showed an average of 9.1 and $21.3 \%$ blebbing in the presence and absence of external NOK-1, respectively. 
tors may share common signalling events that enhance surface nanotubular connections. These events do not involve caspase-8 (Supplementary information, Figure S2) or RIP (our unpublished results) but, similar to other surface changes, may derive from early 'horizontal' signalling mediated by the DISC $[2,25]$

Membrane nanotubes are dynamic plasma membrane extensions that can form channels of communication between neighbouring cells $[8-10,14,15]$. In particular, nanotubes are known to facilitate the transmission of membrane and cytosolic components, organelles [8, 10] and also infective viral particles [14]. However, the membrane nanotubes that have previously been described in T cells appeared to be seamless structures that connect cells without establishing an open communication [14]. We have now discovered that Fas stimulation leads to the formation of another type of tubular connection, which facilitates the bi-lateral transfer of membrane elements (Figures 4 and 5) and also that of cytosolic material between T cells (Figures 4-6). These nanotubular structures are therefore comparable to the tunneling nanotubes that have been reported to be present in many cell types (for reviews, see $[9,10])$. Of note, their formation increases also in fibroblastoid 293T cells after Fas stimulation.

The supporting infrastructure of nanotubes connecting $\mathrm{T}$ cells is constituted by cross-linking actin filaments $[8,9]$, the assembly of which requires Rho GTPases like CDC42 [16]. Cortical actin and its network of associated proteins have been increasingly seen to contribute to the shape changes accompanying Fas signalling $[2,13,25$ 28]. As for Fas-enhanced membrane traffic [13], activation of Rho GTPases lies upstream of the actin-mediated cellular changes that follow Fas stimulation [13, 26, 27], including the surface protrusions forming membrane nanotubes that we show here. Among the GTPases activated by death receptors, CDC42 is likely to play a pivotal role (see Ref. [13] for review). Indeed secramine, a recently described inhibitor of CDC42 [17], blocked the formation of nanotubes and consequent membrane exchanges with comparable efficiency to CdTXB, the general inhibitor of Rho GTPases (Figures 3 and 4). Interestingly, the inhibition of CDC42 and other Rho GTPase enzymes severely curtails the formation of membrane nanotubes and concomitantly reduces the spreading of cell death within populations of Fas-stimulated cells. This suggests that Fas activation of Rho GTPases contributes to the propagation of death signalling not only within cells [13] but also among cells, unveiling a novel social aspect of Fas-induced death.

Homotypic or fratricidal [29] diffusion of death receptor-mediated apoptosis has been previously reported to depend on endogenous molecules like FasL [24]. Here, we show that membrane nanotubes rapidly transmit to neighbouring cells apoptotic signals emanating from Fas, including active caspase- 3 and membrane-bound FasL. Nanotube-mediated transfer of apoptotic signals accounts for the asymmetric rate of death propagation observed in $\mathrm{T}$ cell populations (Figure 1), and therefore may effectively contribute to the spread of fratricidal death before cells autonomously decide to commit suicide. This process would increase the efficiency with which immune responses are abrogated once the antigenic trigger has been eliminated. Consequently, it may have a role in activation-induced cell death [1]. In relation to ALPS, where we demonstrate that communication by nanotubes is defective, it is possible that the incomplete removal of autoreactive bystander $\mathrm{T}$ cells helps to drive and perpetuate $\mathrm{T}$ cell lymphoproliferation and autoimmunity.

How do bystander $T$ cells distinguish between activation signals and signals that induce apoptosis? Cell activation is known to be induced when $\mathrm{T}$ cells are simultaneously exposed to cytokines (e.g., IL-2, IL-13, IL-15) and co-stimulation molecules (e.g., CD28) from antigen-presenting cells and B cells [30]. One explanation may be that homotypic (rather than the usual heterotypic) interaction between $\mathrm{T}$ lymphocytes that we have observed during apoptosis fails to provide the correct costimulatory signals required for survival, i.e., like death by cytokine deprivation or neglect $[1,30]$. Furthermore, if homotypic T-cell interaction after Fas stimulation induces apoptosis of bystander $\mathrm{T}$ cells physiologically, in the future it may be possible to refine this biological mechanism in order to suppress autoreactive $\mathrm{T}$ cells causing autoimmune diseases.

To conclude, our study introduces a new concept in apoptosis, namely the extensive exchange of membrane and cytosolic material between neighbouring $\mathrm{T}$ cells via nanotubes, which promotes fratricide and may consequently contribute to peripheral deletion. We have thus uncovered what may turn out to be the first physiological role for membrane nanotubes in cells vital to the immune system.

\section{Materials and Methods}

\section{Reagents}

FasL was purchased from Apotech/Alexis (Lausanne, Switzerland) and fluorescent probes from Molecular Probes/Invitrogen (Eugene, OR, USA) or Sigma Aldrich (St Louis, MO, USA). Lyophilized HPA and some antibodies were fluorescently conjugated with kits from Molecular Probes [13]. CdTXB was purchased from Calbiochem, z-VAD from Alexis (San Diego, CA, USA) and other reagents from Sigma (St Louis, MO, USA) and Tocris (Bristol, UK). Secramine A came jointly from the Kirchhausen lab (Harvard Medical School, MA, USA) and the Hammond lab (University of Louisville, KY, USA). Antibodies were obtained as follows: $\mathrm{CH}$ - 
11 Fas agonist from Upstate Biotechnology (Lake Placid, WI, USA); allophycocyanin- and FITC-conjugated anti-CD81 (JS81clone) from $\mathrm{BD} /$ Pharmingen (Oxford, UK); PE-conjugated NOK-1 anti-FasL from Biolegend (San Diego, CA, USA); goat anti-FasL from R\&D Systems (Abington, UK); FITC-conjugated anti-CD59 (MEM43 and BRA-10G) from Ancell (Bayport, MN, USA); PE-conjugated anti-CD59 from Leinco (St Louis, MO, USA) and different secondary antibodies from DAKO, Jackson Labs and Molecular Probes.

\section{Cell culture and trasfection}

The human T cell line, Jurkat J6.1 (obtained from European Tissue Collection, Salisbury, UK) and its caspase-8-deficient [31] and RIP-deficient clones were cultured in RPMI medium supplemented with $10 \%$ foetal calf serum, $50 \mathrm{U} / \mathrm{ml}$ penicillin and $50 \mu \mathrm{g} /$ $\mathrm{ml}$ streptomycin in a humidified atmosphere with $5 \% \mathrm{CO}_{2}$ at $37^{\circ} \mathrm{C}$. Activated $\mathrm{CD}^{+} \mathrm{T}$ lymphocytes from healthy donors and ALPS patients were purified by negative selection using the MACS system (Milteny Biotec, Auburn, CA, USA) from peripheral blood lymphocytes isolated with Ficoll centrifugation. Ethics approval and written consent were obtained prior to taking the patient's blood. $\mathrm{T}$ cells were purified after stimulation with either PHA for 3 days or a burst of ionomycin plus PMA [32]. Isolated T cells, whose purity was verified cytometrically, were cultured in RPMI 1640 in the presence of rIL-2 (100 U/ml, PeproTech, London, UK). Human embryonic kidney (293T) cells were cultured in Dulbecco's modified Eagle's medium supplemented with $10 \%$ foetal calf serum and 1\% Penicillin/Streptomycin. These cells were transfected with Transpass D2 Transfection reagent (New England Biolabs, Hitchin, UK) after $24 \mathrm{~h}$ from plating on poly-lysine-coated cover slips within six-well plates. A total of $2 \mu \mathrm{g}$ of cDNA for YFP (empty vector pEYFP-N1 from Invitrogen) or CFP-Histone 2B [33] was added per well and mixed with $1 \mathrm{ml}$ of serum-free medium followed by $4 \mu \mathrm{l}$ of the Transpass reagent. After incubation at room temperature for $20 \mathrm{~min}$, cells were washed with warmed serumfree medium and then incubated with the transfection mixture for $3 \mathrm{~h}$, which was then replaced with $2 \mathrm{ml}$ of full medium for further culture ( $24 \mathrm{~h}$ or more).

\section{Immunocytochemistry}

Cells were washed and resuspended in modified Ringer buffer ( $\mathrm{RB}$, containing $145 \mathrm{mM} \mathrm{NaCl}, 4.5 \mathrm{mM} \mathrm{KCl}, 2 \mathrm{mM} \mathrm{MgCl}, 1 \mathrm{mM}$ $\mathrm{CaCl}_{2}, 5 \mathrm{mM}$ K-Hepes, pH 7.4 and $10 \mathrm{mM}$ glucose) loaded with fluorescent probes for 20-30 min, washed and incubated at 4 to $6 \times$ $10^{6}$ per $\mathrm{ml}$ with recombinant FasL or $\mathrm{CH}-11$ before plating onto cover slips coated with poly-lysine [13, 34]. After adhering for 15-20 min at $37{ }^{\circ} \mathrm{C}$, cells were transferred on ice and then washed with cold PBS before fixation with $4 \%(\mathrm{w} / \mathrm{v})$ paraformaldehyde. For surface staining, fixed cells were treated with diluted fluorescently labelled monoclonal antibodies containing BSA to minimize background. For global immunodetection, fixed cells were permeabilized with $0.4 \%$ Triton-X100 or $0.1 \%$ saponin, blocked with $1 \%$ BSA or serum, incubated with primary antibodies for 15 $30 \mathrm{~min}$, washed again and then incubated with diverse secondary antibodies [13]

\section{Measurement of intercellular membrane exchange}

To measure membrane exchanges by flow cytometry, we used the method of total mean fluorescence subtracted for background
[22] with separate staining of T cells with the red PKH-26 or the mitochondrial marker TMRE and various green probes, including the cytosolic stain CFSE [20]. After mixing in the absence or presence of FasL, cells were analysed with a FACScan ${ }^{\circledR}$ flow cytometer (Becton Dickinson, Palo Alto, CA) using the CellQuest ${ }^{\mathrm{TM}}$ software and appropriate gating from scatter profiles that minimized the inclusion of cell conjugates [35]. Alternatively, mixed cells were seeded on cover slips in the presence of Fas stimulation and then processed for microscopic imaging, as described below.

\section{Fluorescence and time-lapse microscopy}

We routinely imaged cells with DeltaVision $\mathrm{RT}^{\circledR}$ (software Rx. 3.4.3, Applied Precision) at $20{ }^{\circ} \mathrm{C}$ using an automated Olympus IX71 microscope with oil-immersed objectives. Images from stacks of 25-35 sections of $0.2 \mu \mathrm{m}$ were deconvolved for 7-10 cycles and then projected along the z-plane. For time-lapse video microscopy, live cells were incubated with various probes in RB and then seeded at 5 to $6 \times 10^{6}$ per $\mathrm{ml}$ on cover slips coated with poly-lysine (MatteK Corporation, MA, USA). Raw data were acquired after ca. $10 \mathrm{~min}$ of FasL addition within a thermostatted chamber $\left(37^{\circ} \mathrm{C}\right)$, using a Leica DMIRE2 inverted microscope and a high-sensitivity camera (Cascade II, Photometrics). Image .tiff files were analysed after splitting individual 8-bit color data using Image $\mathrm{J}$; subsequently, background and channel cross-talk was eliminated using threshold pixel levels standardized for each probe (for instance, $>21$ out of 255 intensity levels for red HPA). Then the presence of at least two red or green fluorescence spots in cells having predominantly the opposite color was considered sufficient to score cells as 'green with red' and 'red with green', as verified by parallel flow cytometry measurements. The level of uni-lateral membrane exchange was expressed as percentage of the total number of observed cells. Bidirectional exchange via connecting nanotubes was evaluated using the same criteria.

\section{Evaluation of cell death and endocytosis}

Commitment to cell death was evaluated by direct visual inspection of blebbing in live or fixed cells as described [13, 36], integrated with evaluation of nuclear fragmentation after staining with Hoechst 33532 and caspase activation (using the platform of Imagen-Biotech, Manchester, UK). Activation of caspase-8 was measured with a plate reader (Ascent instrument, Thermo, Basingstoke, UK) or cytofluorescence with $10 \mu \mathrm{M}$ Rho-IETD-bis (rhodamine110-carbonyl-Ile-Glu-Thr-Asp-bisamide) [37]. Immunostaining of active caspase- 3 was carried out as reported earlier [13]. For evaluating endocytosis in the same settings used for membrane exchange, we scored the cellular distribution of CD59 [13]. Fluorescent HPA or WGA were also applied for decorating cell surfaces [12].

\section{EM studies}

Cell pellets were fixed with $3.8 \%$ acrolein (TAAB, Aldermaston, UK) plus $2 \%$ paraformaldehyde in $0.1 \mathrm{M}$ phosphate buffer $(\mathrm{pH}$ 7.4) for $1 \mathrm{~h}$ at room temperature. Cells were also processed after attachment onto cover slips as in cytofluorescence experiments, using HRP-conjugated HPA as a membrane marker. Before preparing the final Epon block, cover slip glass was removed with hydrofluoric acid. Ultrathin sections were cut with a diamond knife (Diatome), collected on copper mesh grids and counterstained with uranyl acetate plus lead citrate. They were then examined with a 
Philips Tecnai microscope. Composite electron microscope figures were generated using Adobe Photoshop CS.

\section{Statistical analysis}

Visual analysis of cell morphology was undertaken on sets of at least 50 cells per sample and statistically evaluated with nonparametric tests like Mann-Whitney [38] plus parametric tests like 2-sample $t$-test using the software package MiniTab15 (http:// www.4ulr.com/products/statisticalanalysis).

\section{Acknowledgments}

We thank R Paddon, O Rominiyi and the Bioimaging facility of the University of Manchester for their help. We also thank Dr D Green, R Koshravi-Far, W Malorni, A Viola, D Millar, G Griffiths and I Kimber for helpful discussion. A special thank to H Pelish for providing secramine A. Work in Manchester was supported by BBSRC grant BB/C50846 (UK), while research in Urbino was supported by MIUR-PRIN 2006 (UK).

\section{References}

1 Green DR, Droin N, Pinkoski M. Activation-induced cell death in T cells. Immunol Rev 2003; 193:70-81.

2 Siegel RM, Muppidi JR, Sarker M, Lobito A, Jen M, Martin $\mathrm{D}$ et al. SPOTS: signaling protein oligomeric transduction structures are early mediators of death receptor-induced apoptosis at the plasma membrane. J Cell Biol 2004; 167:735-744.

3 Peter ME, Krammer PH. The CD95(APO-1/Fas) DISC and beyond. Cell Death Differ 2003; 10:26-35.

4 Algeciras-Schimnich A, Shen L, Barnhart BC, Murmann AE, Burkhardt JK, Peter ME. Molecular ordering of the initial signaling events of CD95. Mol Cell Biol 2002; 22:207-220.

5 Rao VK, Straus SE. Causes and consequences of the autoimmune lymphoproliferative syndrome. Hematology 2006; 11:15-23.

6 Martin DA, Zheng L, Siegel RM, Huang B, Fisher GH, Wang J, et al. Defective CD95/APO-1/Fas signal complex formation in the human autoimmune lymphoproliferative syndrome, type Ia. Proc Natl Acad Sci USA 1999; 96:4552-4557.

7 Rieux-Laucat F, Blachere S, Danielan S, De Villartay JP, Oleastro M, Solary E, et al. Lymphoproliferative syndrome with autoimmunity: a possible genetic basis for dominant expression of the clinical manifestations. Blood 1999; 94:25752582 .

8 Rustom A, Saffrich R, Markovic I, Walther P, Gerdes HH. Nanotubular highways for intercellular organelle transport. Science 2004; 303:1007-1010.

9 Davis DM. Intercellular transfer of cell-surface proteins is common and can affect many stages of an immune response. Nat Rev Immunol 2007; 7:238-243.

10 Gerdes HH, Carvalho RN. Intercellular transfer mediated by tunneling nanotubes. Curr Opin Cell Biol 2008; 20:470-475.

11 Blanchard N, Di Bartolo V, Hivroz C. In the immune synapse, ZAP-70 controls $T$ cell polarization and recruitment of signaling proteins but not formation of the synaptic pattern. Immunity 2000; 17:389-399.

12 Degli Esposti M. Organelle intermixing and membrane scrambling in cell death. Methods Enzymol 2008; 422:421-
438.

13 Degli Esposti M, Tour J, Ouasti S, Ivanova S, Matarrese $\mathrm{P}$, Malorni W, et al. Fas death receptor enhances endocytic membrane traffic converging into the golgi region. Mol Biol Cell 2009; 20:600-615.

14 Sowinski S, Jolly C, Berninghausen O, Purbhoo MA, Chauveau A, Kohler K, et al. Membrane nanotubes physically connect $\mathrm{T}$ cells over long distances presenting a novel route for HIV-1 transmission. Nat Cell Biol 2008; 10:211-219.

15 Gurke S, Barroso JF, Hodneland E, Bukoreshtliev NV, Schlicker O, Gerdes HH. Tunneling nanotube (TNT)-like structures facilitate a constitutive, actomyosin-dependent exchange of endocytic organelles between normal rat kidney cells. Exp Cell Res 2008; 314:3669-3683.

16 Wherlock M, Mellor H. The Rho GTPase family: a Racs to Wrchs story. J Cell Sci 2002; 115:239-240.

17 Pelish HE, Peterson JR, Salvarezza SB, Rodriguez-Boulan E, Chen JL, Stamnes M, et al. Secramine inhibits Cdc42dependent functions in cells and Cdc42 activation in vitro. Nat Chem Biol 2006; 2:39-46.

18 Joly E, Hudrisier D. What is trogocytosis and what is its purpose? Nat Immunol 2003; 4:815.

19 Daubeuf S, Puaux AL, Joly E, Hudrisier D. A simple trogocytosis-based method to detect, quantify, characterize and purify antigen-specific live lymphocytes by flow cytometry, via their capture of membrane fragments from antigen-presenting cells. Nat Protoc 2006; 1:2536-2542.

20 Aucher A, Magdeleine E, Joly E, Hudrisier D. Capture of plasma membrane fragments from target cells by trogocytosis requires signaling in T cells but not in B cells. Blood 2008; 111:5621-5628.

21 Veranic P, Lokar M, Schütz GJ, Weghuber J, Wieser S, Hägerstrand $\mathrm{H}$, et al. Different types of cell-to-cell connections mediated by nanotubular structures. Biophys $J$ 2008; 95:44164425.

22 Poupot M, Fournié JJ. Spontaneous membrane transfer through homotypic synapses between lymphoma cells. $J$ Immunol 2003; 171:2517-2523.

23 Blott E J, Bossi G, Clark R, Zvelebil M, Griffiths GM. Fas ligand is targeted to secretory lysosomes via a proline-rich domain in its cytoplasmic tail. J Cell Sci 2001; 114:2405-2416.

24 Herr I, Posovszky C, Di Marzio LD, Cifone MG, Boehler T, Debatin KM. Autoamplification of apoptosis following ligation of CD95-L, TRAIL and TNF-alpha. Oncogene 2000; 19:4255-4262.

25 Muppidi JR, Lobito AA, Ramaswamy M, Yang JK, Wang L, Siegel RM. Homotypic FADD interactions through a conserved RXDLL motif are required for death receptor-induced apoptosis. Cell Death Differ 2006; 13:641-1650.

26 Subauste MC, Von Herrath M, Benard V, Chamberlain CE, Chuang TH, Chu K, Bokoch, et al. Rho family proteins modulate rapid apoptosis induced by cytotoxic T lymphocytes and Fas. J Biol Chem 2000; 275:9725-9733.

27 Parlato S, Giammarioli AM, Logozzi M, Lozupone F, Matarrese $\mathrm{P}$, Luciani F, et al. CD95 (APO-1/Fas) linkage to the actin cytoskeleton through ezrin in human $\mathrm{T}$ lymphocytes: a novel regulatory mechanism of the CD95 apoptotic pathway. EMBO J 2000; 19:5123-5134.

28 Hébert M, Potin S, Sebbagh M, Bertoglio J, Bréard J, Hame- 
lin J. Rho-ROCK-dependent ezrin-radixin-moesin phosphorylation regulates Fas-mediated apoptosis in Jurkat cells. $J$ Immunol 2008; 181:5963-5973

29 Callard RE, Stark J, Yates AJ. Fratricide: a mechanism for T memory-cell homeostasis. Trends Immunol 2003; 24:370-375.

30 Arnold R, Brenner D, Becker M, Frey CR, Krammer PH. How T lymphocytes switch between life and death. Eur J Immunol 2006; 36:1654-1658.

31 Kischkel FC, Lawrence DA, Tinel A, Le Blanc H, Virmani A, Schow $\mathrm{P}$, et al. Death receptor recruitment of endogenous caspase-10 and apoptosis initiation in the absence of caspase-8. $J$ Biol Chem 2001; 276:46639-46646.

32 Legembre P, Daburon S, Moreau P, Moreau JF, Taupin JL. Modulation of Fas-mediated apoptosis by lipid rafts in T lymphocytes. J Immunol 2006; 176:716-720.

33 Upton JP, Valentijn AJ, Zhang L, Gilmore AP. The N-terminal conformation of Bax regulates cell commitment to apoptosis. Cell Death Differ 2007; 14:932-942.

34 Ouasti S, Matarrese P, Paddon R, Khosravi-Far R, Sorice M,
Tinari A, et al. Death receptor ligation triggers membrane scrambling between Golgi and mitochondria. Cell Death Differ 2007; 14:453-461.

35 Horner H, Frank C, Dechant C, Repp R, Glennie M, Herrmann $\mathrm{M}$, et al. Intimate cell conjugate formation and exchange of membrane lipids precede apoptosis induction in target cells during antibody-dependent, granulocyte-mediated cytotoxicity. J Immunol 2007; 179:337-345.

36 Stinchcombe JC, Bossi G, Booth S, Griffiths GM. The immunological synapse of CTL contains a secretory domain and membrane bridges. Immunity 2001; 15:751-761.

37 Packard BZ, Komoriya A, Brotz TM, Henkart PA. Caspase 8 activity in membrane blebs after anti-Fas ligation. J Immunol 2001; 167:5061-5066.

38 Conover WJ. Pratical Nonparametric Statistics. 3rd Edition, New York: John Wiley \& Sons, 1998.

39 Muppidi JR, Siegel RM. Ligand-independent redistribution of Fas (CD95) into lipid rafts mediates clonotypic T cell death. Nat Immunol 2004; 5:182-189.

(Supplementary information is linked to the online version of the paper on the Cell Research website.) 